\title{
Wideband Pass-Band Transmission Line based on Metamaterial-Inspired CPW Balanced Cells
}

\author{
A. L. Borja ${ }^{1}$, A. Belenguer ${ }^{1}$, J. Cascón $^{1}$, H. Esteban ${ }^{2}$, V. E. Boria ${ }^{2}$ \\ ${ }^{l}$ E. U. Politécnica de Cuenca, Universidad de Castilla La Mancha, Campus Universitario, 16071 Cuenca, España \\ ${ }^{2}$ Instituto de Telecomunicaciones y Aplicaciones Multimedia, Universidad Politécnica de Valencia, Camino de Vera s/n, 46022 \\ Valencia, España
}

\begin{abstract}
In this paper, a balanced metamaterial-inspired coplanar waveguide transmission line with enhanced bandwidth is presented. The coplanar waveguide line is composed of a basic cell with two split ring resonators, two series capacitances (interdigital capacitors), two wide shunt inductances (metallic strips) and four shunt capacitances (implemented through openended stubs). The use of split ring resonators together with metallic shunt strips provide negative permeability and permittivity, respectively. Likewise, the employment of additional series and shunt capacitances permits to control both the left- and right-handed bands, thus achieving a balanced composite transmission line. The configuration exhibits a wide band pass response as a result of this balance between advance and delay phase offsets. The interpretation is based on an equivalent circuit model, full-wave electromagnetic analysis, and measured responses of a prototype designed for microwave $(\mathrm{C}$ Band) frequency operation. Due to the small dimensions of the resonators employed, the resulting line is very compact. Potential use of these transmission lines can be foreseen in many applications concerning planar microwave devices with severe size restrictions and wide frequency band pass responses.
\end{abstract}

\section{INTRODUCTION}

In the last years, the possibility of taking advantage of the unusual properties of the so-called metamaterial technology has led to a great deal of research activity. For instance, many efforts have been aimed at reducing the size and improving the performances of, among others, phase-shifters, baluns, couplers, power dividers, and filters [1]. In all cases, the underlying idea for improvement is the achievement of both negative permittivity and permeability in a given frequency range by an appropriate engineering of the dispersion characteristics [2]. At microwave frequencies, a medium which supports backward wave propagation can be fabricated from transmission line loaded with split ring resonators

This work has been supported by Ministerio de Ciencia e Innovación, Spanish Government, under Research Project TEC2010-21520-C04, and by the Autonomous Government of Castilla-La Mancha under Research Project PPII10-0027-1277.

A. L. Borja, A. Belenguer, and J. Cascon are with the Departamento de Ingeniería Eléctrica, Electrónica, Automática y Comunicaciones, Escuela Politécnica de Cuenca, Universidad de Castilla-La Mancha, Campus Universitario, 16071 Cuenca, Spain (email: alejandro.lucas@uclm.es; angel.belenguer@uclm.es; joaquin.cascon@uclm.es). H. Esteban and V. E. Boria are with the Departamento de Comunicaciones, Universidad PolitécnicadeValencia, 46022Valencia, Spain (e-mail: hesteban@dcom.upv.es; vboria@dcom.upv.es).
(SRRs) [1]-[3], and/or reactive elements [4], [5]. Such loading elements enable one to efficiently control the response characteristics simply by adjusting its dimensions and the position. These structures generally incorporate resonant particles such SRRs, open SRRs (OSRRs) and their dual counterparts (i.e. complementary split ring resonator CSRRs or open CSRRs) [6], [7]. Due to the resonant nature of these transmission lines, special efforts have been made to the synthesis of artificial lines exhibiting improved (i.e. wider) bandwidths. A broadband response can be obtained when the right and left-handed behavior coincide. This is known as the balanced composite right/left-handed (CRLH) response. Generally, this kind of periodically loaded line has compact dimensions, due to the electrically small size of the resonators used. It also yields low insertion loss characteristics and a broad fractional bandwidth. Nevertheless, the transmission line usually requires several unit cells (typically between 4 and 5) in order to obtain acceptable selectivity.

This paper present a balanced CRLH transmission line based on a CPW line [8]-[11]. Distributed loading elements are employed in order to provide an improved control of the transmission line response. In total two interdigital capacitors and four shunt open-ended stubs are used. The stubs act as capacitors. The main advantage of the proposed unit cell is the enhanced bandwidth and compact dimensions that can be obtained by varying the loading elements. Section II introduces the configuration of the balanced CRLH transmission line. It also gives an equivalent circuit model for the proposed unit cell. In Section III the results, obtained for a fabricated prototype, are compared with simulations. A number of conclusions are drawn in the final section.

\section{Composite Basic Cell Design ANd Equivalent CIRCUIT MODEL}

The basic cell under study is composed of a highly dispersive coplanar waveguide (CPW) line loaded with semilumped elements. These elements are electrically small compared to the propagating wavelength. In particular, the cell consists of a CPW line loaded with two SRRs, two series capacitances (interdigital capacitors), two shunt inductances (metallic strips) and four shunt capacitances (open-ended stubs). These components are located on the resonators 
symmetry axis. The configuration is depicted in Fig. 1. Bond wires were added in order to avoid the excitation of high order propagating modes. A prototype was fabricated using a mechanical milling machine (i.e. a LPKF Protomat S100 machine). The prototype is shown in Fig. 1b. The substrate material has a thickness of $0.508 \mathrm{~mm}$, permittivity $\left(\varepsilon_{r}\right)$ of 2.2 and loss tangent $(\operatorname{tg} \delta)$ of 0.0009 . The metal utilized is copper having a thickness of $35 \mu \mathrm{m}$, and a conductivity of $5.810^{7}$ $\mathrm{S} / \mathrm{m}$.

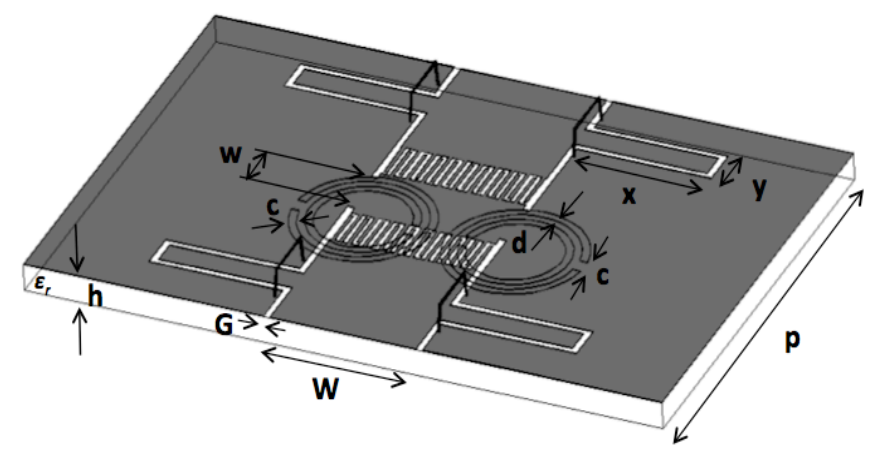

(a)

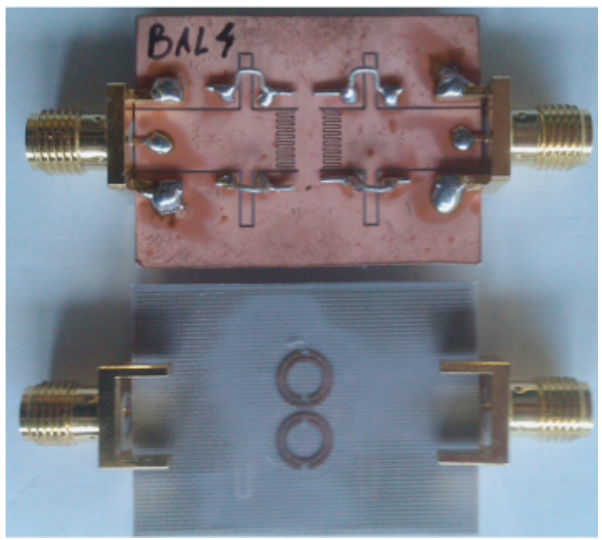

(b)

Fig. 1: (a) Layout of the SRR loaded CPW with shunt strips. (b) Photograph of a fabricated prototype. $\mathrm{CPW}$ dimensions $\mathrm{G}=0.25$ and $\mathrm{W}=5.7$ were chosen to obtain $50 \Omega$ characteristic impedance and avoid a taper transition. Also, basic cell parameters are $\mathrm{p}=25, \mathrm{c}=\mathrm{d}=0.3, \mathrm{w}=2, \mathrm{r}_{\text {int }}=1.8, \mathrm{x}=5, \mathrm{y}=1.5$. All dimensions are in $\mathrm{mm}$.

The presence of SRRs underneath the wide shunt strips provides a double negative effective medium that leads to the presence of a left-handed band pass response above the isolated resonant frequency of the SRR [12]. As it is described, the SSRs provide a negative permeability while shunt strips provide negative permittivity. Likewise, the use of additional series and shunt capacitor permits to control both the left- and right-handed pass bands. In this way it is possible to realize a CRLH, with the aim of achieving a balanced composite transmission line. The four shunt stubs are responsible for lowering the right-handed transmission band, so that if the associated capacitances are chosen properly, the left- and right-handed bands can be obtained at the same frequency. This leads to a balanced composite transmission line with a wideband response.

The lumped element equivalent circuit model for the basic cell can be used to understand the operation. This equivalent circuit is shown in Fig. 2. Due to the symmetry conditions, a magnetic wall has been placed along the centre of the transmission line. Thus, Fig. 2 actually depicts half of a unit cell.

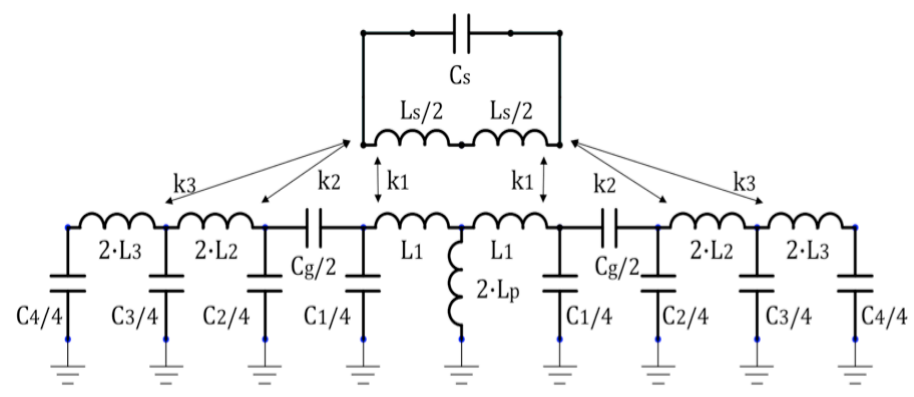

Fig. 2: Basic cell equivalent circuit of the proposed balanced basic cell.

TABLE I. Equivalent circuit element values for the proposed balanced basic cell.

\begin{tabular}{|c|c|c|c|c|c|}
\hline \multicolumn{6}{|c|}{ TABLE I } \\
\hline$C_{s}(\mathrm{pF})$ & $L_{s}(\mathrm{nH})$ & $C_{g}(\mathrm{pF})$ & & $L_{p}(\mathrm{pH})$ & $L_{l}(\mathrm{nH})$ \\
\hline 0.53 & 1.25 & 0.66 & & 5 & 0.46 \\
\hline$L_{2}(\mathrm{nH})$ & $L_{3}(\mathrm{nH})$ & $C_{l}(\mathrm{pF})$ & & $C_{2}(\mathrm{pF})$ & $C_{3}(\mathrm{pF})$ \\
\hline 0.96 & 0.13 & 0.56 & & 0.43 & 2.1 \\
\hline $\begin{array}{c}C_{4}(\mathrm{pF}) \\
0.12\end{array}$ & & $\begin{array}{c}k_{1} \\
0.55\end{array}$ & $\begin{array}{c}k_{2} \\
0.14\end{array}$ & $\begin{array}{c}-\quad k_{3} \\
0.05\end{array}$ & \\
\hline
\end{tabular}

The model employed for the different portions of the CPW transmission line is based upon a simple LC circuit. Thus, the equivalent components $L_{1}, C_{1}, L_{2}, C_{2}, L_{3}$, and $C_{4}$ are the persection inductance and capacitance of the portion lines placed between both interdigital capacitors, between interdigital capacitors and the stubs, and at input/output access ports. The series inductances and the shunt capacitances mentioned above are defined from the geometry of the CPW transmission line (see for instance [13]). $C_{3}$ models the shunted capacitance, which is implemented by means of the openended stubs. The interdigital capacitor is modelled by the series element $C_{g} . L_{p}$ models the inter-connection to the lateral ground planes by means of wide shunt strips. Equally, the SRRs are represented by means of an $L_{s}-C_{s}$ resonant circuit. A transmission-line model can be used to determine the capacitance $C_{s}$ and the inductance $L_{s}$. This assumes that the metallic strips are described as a pair of coplanar strips, as it was proposed by Marques in [14]. Inter-resonator coupling can be neglected since the SRRs are circular in shape [15], thus reducing the interaction between them. Besides, only a single cell is considered. The calculation of $\mathrm{L}$ and $\mathrm{C}$ relies on an assumption about the substrate permittivity. To verify this assumption the authors have conducted a full-wave 
simulation. The simulation shows that the electric field lines are mostly contained in the dielectric substrate, thus justifying the use of $\varepsilon=\varepsilon_{\text {eff. }}$. We assumed that the resonators are magnetically coupled to the three regions of the host transmission line. Therefore, the coupling coefficients $k_{1}, k_{2}$ and $k_{3}$ account for the interaction between the resonators and the host transmission line. The coupling coefficient $k_{l}$ is determined from the mutual inductance, according to the fractional theory, described in [2]. As pointed out, the mutual inductance $M$ and the coupling coefficient $k_{l}$ can be obtained by using the following equations:

$$
\begin{aligned}
& M=f L_{1} \\
& k_{1}=\frac{M}{\sqrt{L_{1} L_{s} / 2}}
\end{aligned}
$$

where $f$ is the fraction of the slot area occupied by the resonators. However, the coefficient $k_{l}$ has been slightly tuned since the shunt strips and the interdigital capacitors modify the coupling level between the line and the rings. Consequently, the initial value, determined from the fractional theory, has been tuned slightly according to the results of a parametric analysis. Also, the coefficients $k_{2}$ and $k_{3}$ were adjusted by means of a curve fitting procedure.

\section{SIMULATION AND EXPERIMENTAL RESULTS}

In this section, we present the frequency response of the balanced unit cell. This unit cell was designed for operation at C-band frequencies. Fig. 3 illustrates the results obtained from full-wave and circuital simulations. These simulations were performed using Ansoft HFSS and Ansoft Designer, respectively. They were also verified by performing measurements using a prototype.

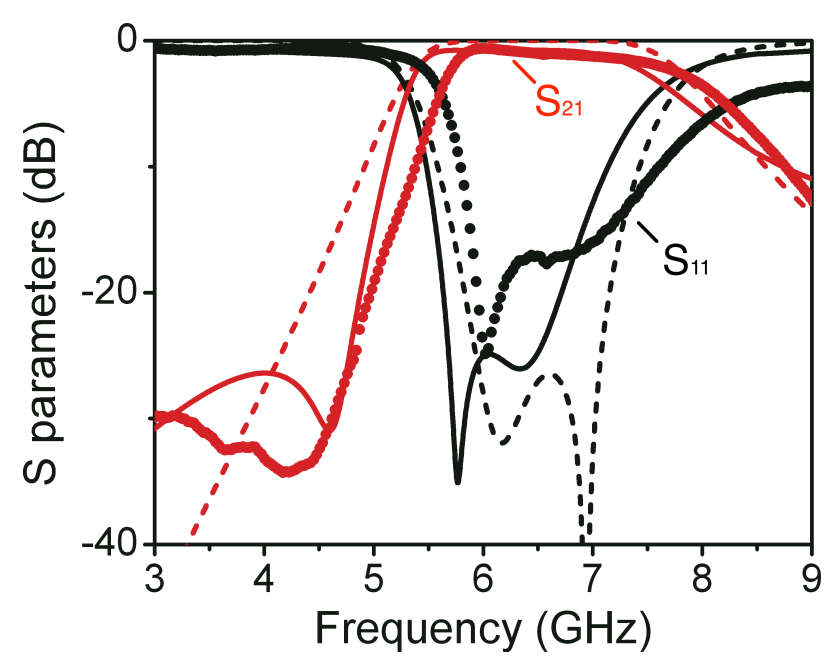

Fig. 3: Simulated (solid line), equivalent circuit (dashed line) and measured (solid symbol) reflection $\mathrm{S}_{11}$ (black) and transmission $\mathrm{S}_{21}$ (red) coefficients for the proposed line depicted in Fig. 1.
Measurements have been performed with an Anritsu vector network analyzer calibrated with a Through-Open-ShortMatch kit. These measurements were performed in the frequency band from $3 \mathrm{GHz}$ to $8 \mathrm{GHz}$. A wide transmission band is obtained, as confirmed by inspecting $S_{11}$ and $S_{21}$. The measured transmission line response exhibits a band pass centred around $6.6 \mathrm{GHz}$ with a transmission zero close to 4.3 $\mathrm{GHz}$. This central frequency is above the SRR resonance which can be estimated as follows: $\mathrm{f}=(2 \pi)^{-1}\left(L_{s} C_{S}\right)^{-1 / 2}$. For the isolated resonator $\mathrm{f}=5.8 \mathrm{GHz}$. The structure yields a fractional bandwidth (FBW) of $32.5 \%$ and an insertion loss below 2.5 $\mathrm{dB}$ throughout the entire transmission band. In this regard, unbalanced left-handed band pass filters typically yield a FBW between $2 \%-8 \%$ [10]. In spite of this, it has been demonstrated that a larger bandwidth (even with FBW close to $110 \%$ ) can be achieved by employing other structures and technologies [16]. Moreover, two poles can be observed in the reflection coefficient corresponding to each left- and righthanded band. Concerning selectivity, there is clearly a dip in the transmission (i.e. $\mathrm{S}_{21}$ curve) response at approximately 4.3 $\mathrm{GHz}$. However, the level of pass band rejection at $8.5 \mathrm{GHz}$ is around $10 \mathrm{~dB}$. It is worth mentioning that the frequency response characteristics correspond to a single unit cell. The selectivity can be improved by simply cascading several unit cells [10]. However an optimization process should be carried out in order to minimize the pass band ripple caused by the interaction between adjacent cells. Additionally, higher levels of pass band insertion loss are expected. At last, a good agreement between simulated and experimental results can be found. Possible discrepancies are attributed to fabrication tolerances and measurements inaccuracies.

In order to have a deeper understanding of the mechanism for wave propagation along the line, let us now consider the dispersion characteristics. The phase constant $\beta$ has been calculated from Eq. 3 as described in Ref. [5].

$$
\beta p=\cos ^{-1}\left(\frac{1-S_{11} S_{22}+S_{12} S_{21}}{2 S_{21}}\right)
$$

The dispersion diagram was obtained from the full-wave simulated and measured scattering parameters, shown in Fig. 4. From the analysis of the simulation results it is clear that backward wave transmission (phase decreases from $5 \mathrm{GHz}$ to $5.8 \mathrm{GHz}$ ) is switched to forward wave transmission (phase increases from $5.8 \mathrm{GHz}$ to $8 \mathrm{GHz}$ ) within the region of the transmission band close to the SRR resonance. Additionally there is a phase offset that occurs close to the anti-resonance at $8.3 \mathrm{GHz}$. However, some quantitative discrepancies can be observed in the experimental results, which can be mainly attributed to fabrications and substrate tolerances, as well as to the bond-wire connections. The inaccuracies in the fabricated prototype lead to a slight unbalance between the phases of the left- and high-handed modes. These errors could be minimized by improving the tolerance of the milling process or using a laser fabrication processes. 


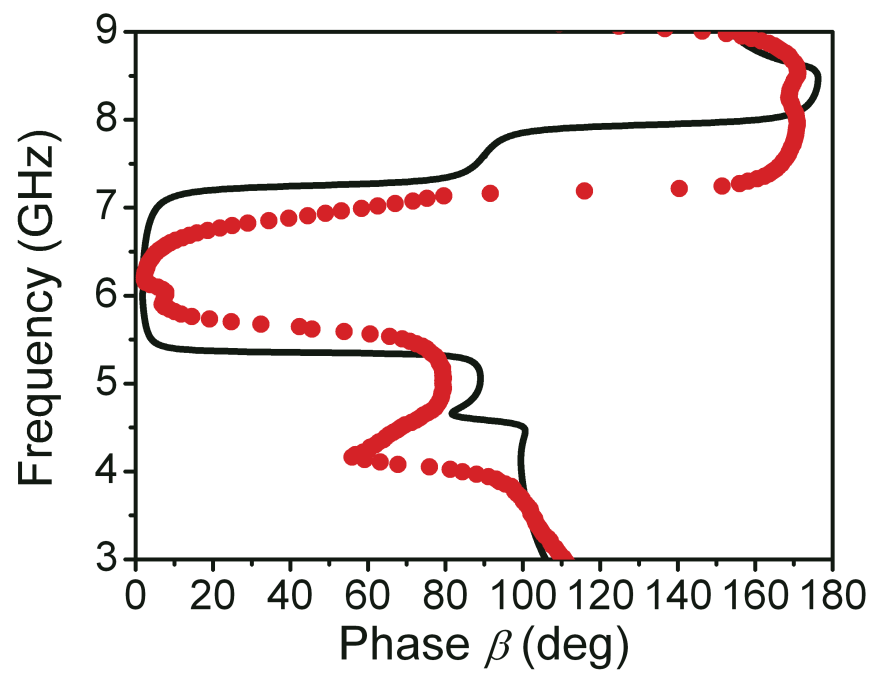

Fig. 4: Simulated (solid black line) and measured (solid red symbol) dispersion diagram of the balanced basic cell.

\section{CONCLUSIONS}

This letter presents a balanced coplanar waveguide transmission line loaded with split ring resonators, series interdigital capacitors, wide shunt metallic strips and shunt open-ended stubs. Left and righted modes of propagation are obtained at the same frequency. Consequently the configuration exhibits a wide band pass response. The paper therefore demonstrates a technique for achieving a considerable increase in the bandwidth of these highly dispersive transmission lines. Specifically, a FBW of around $33 \%$ can be achieved with an acceptable tradeoff between the insertions loss and selectivity. Finally, a confirmation of the balanced behavior has been obtained by studying the dispersion characteristics of the basic unit cell. The proposed structure offers an alternative method for designing planar frequency filtering structures in applications where wide pass band responses with compact dimensions are needed.

\section{ACKNOWLEDGEMENT}

This work has been supported by Ministerio de Ciencia e Innovación, Spanish Government, under Research Project TEC2010-21520-C04, and by the Autonomous Government of Castilla-La Mancha under Research Project PPII10-00271277.

\section{REFERENCES}

[1] R. Marques, F. Martin, and M. Sorolla, Metamaterials with Negative Parameters: Theory, Design and Microwave Applications, John Wiley \& Sons, 2008.

[2] F. Martin, J. Bonache, F. Falcone, M. Sorolla, and R. Marques, "Split ring resonator-based left-handed coplanar waveguide," Applied Physics Letters, vol. 83, no. 22, p. 4652-4654, 2003.

[3] J. Bonache, I. Gil, J. García-García, and F. Martín, "Novel microstrip bandpass filters based on complementary split-ring resonators," IEEE Trans. Microw. Theory Tech., vol. 54, no. 1, pp. 265-271, Jan. 2006.

[4] G. V. Eleftheriades, A. K. Iyer, and P. C. Kremer, "Planar negative refractive index media using periodically L-C loaded transmission lines," IEEE Trans. Microw. Theory Tech., vol. 50, no. 12, pp. 2702-2712, Dec. 2002.
[5] C. Caloz and T. Itoh, Electromagnetic Metamaterials: Transmission Line Theory and Microwave Applications, Wiley, New York, 2005.

[6] M. Gil, J. Bonache, J. Garcia-Garcia, J. Martel, and F. Martin, "Composite right/left-handed metamaterial transmission lines based on complementary split-rings resonators and their applications to very wideband and compact filter design," IEEE Transactions on Microwave Theory and Techniques, vol. 55, no. 6, pp. $1296-1304$, 2007.

[7] A. Velez, F. Aznar, J. Bonache, M. C. Velazquez-Ahumada, J. Martel, and F. Martin, "Open complementary split ring resonators (OCSRRs) and their application to wideband CPW band pass filters," IEEE Microwave and Wireless Components Letters, vol. 19, no. 4, pp. 197 199, 2009.

[8] A. L. Borja, J. Carbonell, V. E. Boria, and D. Lippens, "Symmetrical frequency response in a split ring resonator based transmission line," Applied Physics Letters, vol. 93, p. 203505, Nov. 2008.

[9] A. L. Borja, J. Carbonell, V. E. Boria, and D. Lippens, "Highly selective left-handed transmission line loaded with split ring resonators and wires," Applied Physics Letters, vol. 94, no. 14, 2009.

[10] A. L. Borja, J. Carbonell, V. E. Boria, J. Cascon., and D. Lippens, "A $2 \%$ bandwidth c-band filter using cascaded split ring resonators," IEEE Antennas and Wireless Propagation Letters, vol. 9, pp. 256-259, 2010.

[11] J. Carbonell, A. L. Borja, V. E. Boria, and D. Lippens, "Duality and superposition in split ring resonator loaded planar transmission lines," IEEE Antennas and Wireless Propagation Letters, vol. 8, pp. 886-889, 2009.

[12] F. Aznar, J. Bonache and F. Martin, " Improved circuit model for lefthanded lines loaded with split ring resonators," Applied Physics Letters, vol. 92, p. 043512, 2008.

[13] Mongia, R., Bahl, I., and Bhartia, P.: 'RF and microwave coupled-line circuits' (Artech House, Boston, 1999).

[14] R. Marques, F. Mesa, J. Martel, and F. Medina: "Comparative analysis of edge- and broadside-coupled split ring resonators for metamaterial design-theory and experiments', IEEE Transactions on Antennas and Propagation, vol. 51, pp. 2572-2581, 2003.

[15] I. Gil, J. Bonache, M. Gil, J. García-García, F. Martín, and R. Marqués, "Accurate circuit analysis of resonant-type left handed transmission lines with inter-resonator coupling", Journal of Applied Physics, vol. 100 , p. 074908, 2006.

[16] H. V. Nguyen and C. Caloz, "Broadband highly selective bandpass filter based on a tapered coupled-resonator (TCR) CRLH structure" Proc. of the European Microwave Association, vol. 2, pp.44-51, 2006. 